\title{
Koronarstenosen
}

\section{Fraktionelle Flussreserve optimiert den Ischämienachweis}

\section{Das Ausmaß einer Ischämie determi- niert letztlich die Prognose bei Ver- engungen der Koronargefäße. Eine erfolgreiche Intervention mit dem Ziel einer funktionellen Verbesserung setzt daher streng genommen immer einen Nachweis einer relevanten Ischämie voraus.}

Belastungs-EKG oder präinterventionelle Bildgebung mit Stress-Echokardiografie, Stress-MRT oder PET sind entweder zu ungenau, nicht verfügbar oder den Patienten nicht zuzumuten, erklärte Dr. Waldemar Bojara, Koblenz. Auch mit der Koronarangiografie oder intravaskulärem Ultraschall (IVUS) ist die Hämodynamik intermediärer Stenosen oft nicht abzuschätzen. Die invasive Bestimmung der fraktionellen Flussreserve (FFR) mit einem Druckdraht (Pressure Wire ${ }^{\mathrm{TM}}$ Certus) ist nach den 2-Jahres-Daten der FAME-Studie insbesondere für Patienten mit stabiler Belastungskoronarinsuffizienz, mit mäßiggradigen Stenosen sowie mit Mehrgefäßerkrankungen vorteilhaft.

Im prospektiven randomisierten Vergleich von FFR und Angiografie bei Patienten mit Mehrgefäßerkrankungen reduzierte die FFR-Messung während der Intervention im Vergleich zur Angiografie nach zwei Jahren die Raten von Tod oder Myokardinfarkt um $34 \%$ und das Auftreten von Myokardinfarkten um $37 \%$. Zugleich wurden in der FFRGruppe im Mittel nur 1,9 Stents, in der AngioGruppe dagegen 2,7 Stents implantiert.

Die FFR nutzt jedoch nicht nur dem Gesundheitssystem sondern auch der einzelnen Klinik, ergänzte Priv.-Doz. Dr. Johannes Rieber aus München. Ein erhöhtes Entgelt im DRG-System lasse sich mit der durch den OPS-Code 1-279.a kodierten Methode erzielen, wenn sie mit der Koronarangiografie während eines mehr als zweitägigen Klinikaufenthalts oder mit einer PCI bei Implan- tation genau eines Stents kombiniert werde. Eine DRG-Simulation von knapp 25000 Fällen aus 100 deutschen Kliniken mit Einsatz eines Herzkatheters ergab, so Rieber, dass der durchschnittliche Erlös aller Fälle dann von 2490 auf 3522 Euro stieg, was eine kostendeckende Messung ermöglichte.

Die überragende Bedeutung der FFR bei der Beurteilung der hämodynamischen Relevanz von Stenosen spiegelt sich auch in den aktuellen ESC/EACTS-Leitlinien zur myokardialen Revaskularisierung wider, erklärte Prof. Volker Schächinger aus Fulda. Demnach gilt für die FFR eine Klasse-la-Empfehlung, „wenn keine objektiven Hinweise für eine Ischämie vorhanden sind".

(hae) II

II Jahrestagung der Deutschen Gesellschaft für Kardiologie, Mannheim, April 2011 (Veranstalter: St. Jude Medical)

\section{Therapie von Vorhofflimmern Antiarrhythmikum bewährt sich in der Praxis}

Seit $\mathbf{5 0 0}$ Tagen ist das Antiarrhythmikum Dronedaron in Deutschland für die Therapie des Vorhofflimmerns zugelassen. Erste praktische Erfahrungen bestätigen das günstige Nutzen-Risiko-Verhältnis dieser Substanz.

„Vorhofflimmern ist keine harmlose EKG- Anomalie, sondern es erhöht das Risiko für Schlaganfall, Herzinfarkt und Tod, d.h. es steigert das Risiko für die kardiovaskuläre Morbidität und Mortalität", sagte Prof. Hendrik Bonnemeier, Kiel. Mit Dronedaron (Multaq ${ }^{\circledR}$ ) steht ein Antiarrhythmikum zur Verfügung, das bei den betroffenen Patienten auch die Hospitalisierungsrate senkt, wodurch die antiarrhythmische Therapie einen neuen Stellenwert erhält.

Die Vermeidung eines Krankenhausaufenthalts hat als Therapieziel auch Einzug in die neuen ESC-Leitlinien für die Therapie des Vorhofflimmerns gehalten. Ausschlagge-

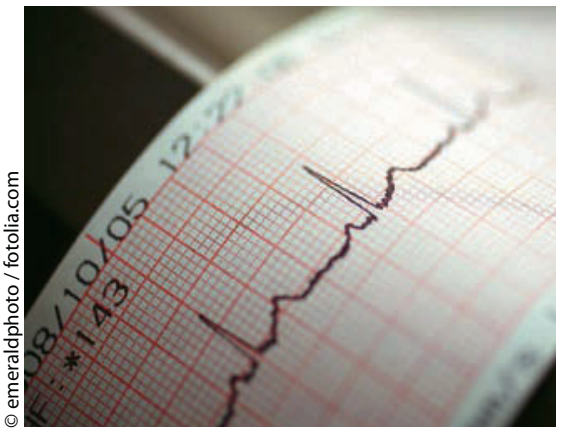

Bei Vorhofflimmern ist neben dem Herzrhythmus auch das Schlaganfallrisiko bedeutsam.

bend hierfür waren die Ergebnisse der ATHENA-Studie. In dieser Studie konnte erstmals für ein Antiarrhythmikum (nämlich Dronedaron) dokumentiert werden, dass es bei Patienten mit Vorhofflimmern den kombinierten Endpunkt aus Mortalität und Hospitalisierung günstig beeinflusst. Auch zeigt Dronedaron im Vergleich zu anderen Anti- arrhythmika, die bei Vorhofflimmern eingesetzt werden, weniger Nebenwirkungen, insbesondere treten gefährliche Proarrhythmien seltener auf. Davon profitieren insbesondere KHK-Patienten, bei denen Klasse-IAntiarrhythmika kontraindiziert sind.

In einer neuen Subgruppenanalyse zeigte sich, dass Dronedaron das Schlaganfallrisiko um 34\% senkt.„Vieles spricht dafür, dass Dronedaron über seine antiarrhythmische Wirksamkeit hinaus auch pleiotrope Effekte entfaltet, vor allem im Hinblick auf eine Verbesserung der Mikrozirkulation", so Prof. Andreas Götte, Paderborn.

Zwischenzeitlich konnte mit Dronedaron, das vor 500 Tagen in Deutschland zugelassen wurde, erste praktische Erfahrung gesammelt werden.,„Diese bestätigen das günstige Nutzen-Risiko-Verhältnis der Studiendaten", so Priv.-Doz. Ralph Bosch, Ludwigsburg. Günstig bei dieser Substanz sei, dass es neben seiner antiarrhythmischen auch eine 